Journal of Animal and Veterinary Advances 11 (16): 2890-2897, 2012

ISSN: $1680-5593$

(C) Medwell Journals, 2012

\title{
Expression of Human Granulocyte-Macrophage Colony-Stimulating Factor in Stably-Transformed BmN and Sf-9 Cells and Silkworms by a Non-Transposon Vector
}

\author{
${ }^{1}$ HaoKun Zhang, ${ }^{1,2}$ GuangLi Cao, ${ }^{1}$ YanMei Li, ${ }^{1,2}$ Ren Yu Xue and ${ }^{1,2}$ ChengLiang Gong \\ ${ }^{1}$ School of Biology and Basic Medical Science, \\ ${ }^{2}$ National Engineering Laboratory for Modern Silk, Soochow University, \\ 215123 Suzhou, P.R. China
}

\begin{abstract}
This study aimed to explore the possibility of non-transposon vector mediated foreign gene expression in cultured insect cells and transgenic silk worms. To this end, the human Granulocyte-Macrophage Colony-Stimulating Factor $(h G M-C S F)$ gene was inserted into the insect cell expression vector pIZT-V5-His to generate the recombinant vector pIZT-hGM-CSF. After transfection of BmN and Sf-9 cells with the pIZThGM-CSF vector, stably-transformed cells expressing the $h G M-C S F$ gene were selected using the antibiotic zeocin at a final concentration of $300-400 \mu \mathrm{g} \mathrm{mL}{ }^{-1}$. Expression of a $22 \mathrm{kDa}$ protein band representing hGM-CSF was detected in the transformed cells by sodium dodecyl sulfate-polyacrylamide gel electrophoresis and Western blotting. The expression levels of hGM-CSF in BmN and Sf-9 cells were determined by Enzyme-Linked Immunosorbent Assay (ELISA) to be about 0.7 and $0.3 \mathrm{ng} / 10^{6}$ cells, respectively. The transgenic vector pIZT-hGM-CSF was transferred into silkworm eggs using sperm-mediated gene transfer. Transgenic silkworms were obtained after screening for the $g f p$ gene and were verified by polymerase chain reaction, dot hybridization

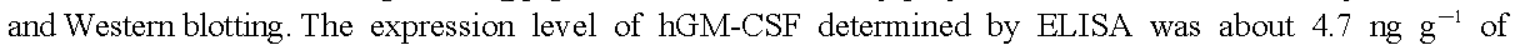
freeze-dried silk glands in the G5 generation. These results suggest that heterologous genes can be integrated into cultured $\mathrm{BmN}$ and $\mathrm{Sf}-9$ cells and into the silkworm genome using a non-transposon vector and can be expressed successfully.
\end{abstract}

Key words: Non-transposon vector, BmN cells, Sf-9 cells, Bombyx mori, hGM-CSF, transgene

\section{INTRODUCTION}

Lepidopteran cell lines have a wide range of applications in the production of recombinant proteins, identification of gene functions and in studies of baculoviruses and the analysis of apoptosis in insect cells (Wickham et al., 1992). Insect cells are often used with the Baculovirus Expression Vector System (BEVS) for the large-scale production of recombinant proteins. However because the host cells are killed during each infection cycle, recombinant protein expression is transient and production can only be achieved in batches (Zhang et al., 1993). In addition, proteins expressed inbaculovirusinfected cells are often incompletely processed (Harrison and Jarvis, 2007) and may thus be unsuitable for in vivo applications (Grossmann et al., 1997). Attempts to overcome this problem have led to the development of a stable, Plasmid-Based Expression System (Jarvis et al., 1990; Jarvis and Finn, 1996; Joyce et al., 1993;
Henderson et al., 1995) capable of continuous protein production in Spodoptera frugiperda (Sf9) cells. However, although sever al insect cell lines have been stably transformed there have been few reports of the use of Bombyx mori cells (Xue et al., 2009). Several types of expression vectors including transpos able-elementderived vectors (Mandrioli and Wimmer, 2003; Xue et al., 2009) site-specific gene-integration vectors and the pIZT/V5-His vector (In vitrogen) (Lu et al., 2011) have been used for the stable transformation of insect cell lines. The $\mathrm{pIZT/V5-His} \mathrm{vector} \mathrm{was} \mathrm{developed} \mathrm{for} \mathrm{the} \mathrm{stable}$ expression of exogenous genes in Lepidopteran cells and use the immediate-early 2 (OpIE2) promoter from Orgyia pseudotsugata Nucleopolyhedrovirus (OpNPV) to control exogenous gene expression with a zeocin-green fluorescent protein (gfp )fused gene as a selective marker.

The silkworm (B. mori) has been domesticated and is currently rearedon a large scale. It is both economically important and also represents a useful model for research

Corresponding Author: ChengLiang Gong, School of Biology and Basic Medical Science, Soochow University, 215123 Suzhou, China 
silkworms have been developed as bioreactors for the production of recombinant proteins using BEVS and transgenes. However, the former is a transient expression system in which the silkworm dies as a result of viral infection and continuous expression of the exogenous gene in the offspring cannot be achieved. New strategies are therefore needed to enable the expression of exogenous genes in silkworms.

Transfer of an exogenous gene into silkworm genomic DNA is a promising strategy for expressing recombinant proteins and several target genes including prolyl hydroxylase $\alpha$-subunit and human collagen (Takahiro et al., 2006), human basic fibroblast growth factor (Hino et al., 2006), human serum albumin (Ogawa et al., 2007), feline interfer on (Kurihara et al., 2007), human insulin-like growth factor-I (Zhao et al., 2009) and human Granulocyte-Macrophage ColonyStimulating Factor (GM-CSF) have already been expressed successfully in transgenic silkworms. Gene transfer to produce transgenic silkworms is usually mediated by transposon vectors (Tamura et al., 1997; Uchino et al., 2007) or homologous recombination vectors (Yamao et al., 2009). However, although transgenic silkworms can be generated using the above approaches, few studies have reported the generation of transgenic silkworms mediated by non-transposon vectors and non-homologous recombination vectors (Cui et al., 2011).

Human GM-CSF (hGM-CSF) is a glycoprotein involved in the regulation of hematopoiesis and immunoregulation. To date, recombinant hGM-CSF has been successfully expressed in transformed cells of Escherichia coli, yeast, tobacco, insects (moths and silkworms) and mammals (COS cells, mice and rats) (Burgess et al., 1987; Miyajima et al., 1986; Aua et al., 1996; Shi et al., 1996; Wong et al., 1985; Tamura et al., 1997; Ryoo et al., 2001; Kodaira et al., 1997).

In this study, researchers explored the possibility of non-transposon-vector-mediated foreign gene expression in cultured insect cells and transgenic silkworms. The $h G M-C S F$ gene was inserted into insect cells using the expression vector $\mathrm{pIZT/V5-His} \mathrm{to} \mathrm{generate}$ the recombinant vector pIZT-hGM-CSF. This vector was used to transform BmN and $\mathrm{Sf}-9$ cells as well as silk worms. The results of this study suggest that heterologous genes can be integrated into the genome of cultured insect cells and silkworms using a nontransposon vector and can be expressed successfully.

\section{MATERIALS AND METHODS}

Construction of recombination transgenic transgenic vector PIZT-hGM-CSF: The hGM-CSF fragment amplified from the plasmid pSK-IE-hGM-CSF (Cao et al., 2006) using the primers hGM-CSF-1 (5'-cgg gta cca tgt ggc tgc aga gcc tgc-3', KpnI site is underlined) and hGM-CSF-2 (5'-cgg aat tcc act cet gga ctg gct ccc-3', EcoRI site is underlined) was digested with $\mathrm{KpnI} / \mathrm{EcoRI}$ and ligated in to the pIZT/V5-His vector (Invitrogen, Frederick, MD,USA) to generate the plasmid pIZT-hGM-CSF. This plasmid contained a zeocin-resistance gene fused with a green fluorescent protein $(g f p)$ gene driven by the OpNPV ie-1 promoter and the $h G M-C S F$ gene controlled by the OpNPV IE2 promoter.

Cellculture and transfection: The $\mathrm{BmN}$ and $\mathrm{Sf}-9$ cell lines were derived from Lepid opteran insects. They were cultured in TC-100 medium (Invitrogen) at $27^{\circ} \mathrm{C} . \mathrm{BmN}$ and Sf-9 cells $\left(1 \times 10^{6}\right)$ were transfected with $2 \mu \mathrm{g}$ of the plasmid using Lipofectamine 2000 (Roche, Mannheim, Germany). $\mathrm{BmN}$ and $\mathrm{Sf}-9$ cells were screened $48 \mathrm{~h}$ post-transfection with zeocin at a final concentration of $300-400 \mu \mathrm{g} \mathrm{mL} L^{-1}$ for 8 weeks. Stably-transformed BmN and Sf-9 cells were subsequently cultured with medium containing zeocin $\left(50 \mu \mathrm{gL}^{-1}\right)$.

Introduction of foreign gene into silkworm eggs: Spermmediated gene transfer was performed according to Zhao et al. (2009). A total of 1.5-2.0 $\mu \mathrm{L}$ of the transgenic vector pIZT-hGM-CSF at a concentration of $3 \mu \mathrm{g} \mu \mathrm{L}^{-1}$ was injected into the copulatory pouch of copulated female moths. After spawning and egg incubation, silkworms were reared on mulberry leaves at $25^{\circ} \mathrm{C}$.

Screening and identification of transgenic silkworms: G0 transgenic silkworms were observed under a stereomicroscope (Olympus SZX12, Tokyo, Japan) and fluorescent individ uals were fed with normal mulberry leaves. Genomic DNA was extracted from moths with green fluorescence and identified by Polymerase Chain Reaction (PCR) using hGM-CSF primers. Genomic DNA was extracted from a fluorescent moth of the G5 generation, denatured by boiling and dotted onto anylon membrane. DNA hybridization with a DIG-labeled gfp probe, membrane washing and signal detection were carried out using a DIG DNA labeling and detection kit (Roche) according to the manufacturer's instructions. Genomic DNA from a normal silkworm and the plasmid pIZT-hGM-CSF were used as negative and positive controls, respectively.

Extraction of protens from Posterior Silk Glands (PSGs): PSGs dissected from transgenic silkworms were freezedried and powdered. The powder was dissolved in tissue lysis buffer (glycerin 10\%, v/v, Sodium Dodecyl Sulfate 
(SDS) $2.5 \%, \mathrm{w} / \mathrm{v}$, mercaptoethanol $5 \%, \mathrm{v} / \mathrm{v}$, Tris-Cl $625 \mathrm{mmol} \mathrm{L}^{-1}, \mathrm{pH} 6.8$ ) and gently shaken for $12 \mathrm{~h}$ at $0^{\circ} \mathrm{C}$ followed by centrifugation at $12,000 \mathrm{~g}$ for $10 \mathrm{~min}$. The supernatant was then collected for SDS-Polyacrylamide Gel Electrophoresis (PAGE), Western blotting and Enzyme-Linked Immuno Sorbent Assay (ELISA).

SDS-PAGE and Western blotting: Transfo rme $\mathrm{d} B \mathrm{BmN}$ and Sf-9 cells were suspended in $0.65 \% \mathrm{NaCl}$ and precipitated in ethanol. Both the cell proteins and the proteins extracted from the PSGs were boiled with an equal volume of $2 \times \mathrm{SDS}$ loading buffer for $5 \mathrm{~min}$ at $100^{\circ} \mathrm{C}$ and separated electrophoretically on $15 \%$ polyacrylamide gradient gels. The gels were either stained with Coomassie Brilliant Blue 250 or used for immuno blotting analysis. The proteins on the gel were transferred to polyvinylidene fluoride membranes which were then reacted with rabbit anti-hGM-CSF (Boster, Wuhan, China), goat anti-Rb (Boster) and 3, 3-diamo nobenzidine tetrahydrchloride (DAB).

Detection of hGM-CSF expression: To determine the expression levels of the $h G M-C S F$ gene in the transforme $\mathrm{d} \mathrm{BmN}$ and Sf-9 cells, the cells were screened with zeocin for 3 months, after which about $80 \%$ displayed green fluorescence. The transformed-cell suspension $\left(5 \times 10^{5}\right.$ cells $\mathrm{mL}^{-1}$ medium) was maintained in aniced-water bath and treated with ultrasonication, followed by centrifugation at $4^{\circ} \mathrm{C}$ to isolate the supernatant. Control cells were treated in the same way. The expression levels of hGM-CSF were determined using an ELISA kit (Boster) according to the manufac turer's protocol.

\section{RESULTS}

Identification of recombinant vectors: The $h G M-C S F$ gene fragment amplified from pSK-IE-hGM-CSF was inserted into the non-transposon vector $\mathrm{pIZT/V5-His} \mathrm{to}$ generate vector pIZT-hGM-CSF in which azeocinresistance gene fused with the $g f p$ gene was driven by the OpNPV IEl promoter and the $h G M-C S F$ gene was controlled by the OpNPV IE2 promoter. The vector construct was examined by $\mathrm{PCR}$ using primer pairs hGM-CSF-1/hGM-CSF-2. A PCR product of $0.43 \mathrm{~kb}$ corresponding to the antic ipated size was detected, suggesting that pIZT-hGM-CSF had been constructed successfully.

Screening and identification of transformed cells: About $10 \%$ of the cells emitted green fluorescence at $48 \mathrm{~h}$ after transfection with $\mathrm{pIZT/V5-His-hGM-CSF}$, suggesting that the vector had entered the cells resulting intransient expression of the zeocin-gfp fused gene. The transfected cells at $48 \mathrm{~h}$ post-transfection were screened continuously with zeocin and the increasing prolife ration of fluorescent cells indicated that they were resistant to zeocin as a result of expression of the zeocin-resistance gene.The proportion of green fluorescent cells reached $80 \%$ after screening for 1 month and obviously different fluorescence intensities were observed in individual cells. (Fig. 1a-d). Selection with zeocin for a further month did not greatly increase the proportion of fluorescent cells. The transfected cells with a stable percentage of green fluorescent cells were further cultured for $>1$ year.

To verify the stable integration of pIZT-hGM-CSF into the genome, genomic DNA was extracted from stablytransformed BmN and Sf-9 cells with or without GFP expression. The anticipated PCR products corresponding in size to hGM-CSF $(0.43 \mathrm{~kb}$ ) (Fig. 1e) were detected in the transfected cells where as no PCR products were recovered from the control cells. These results strongly suggest that the transformation of the $\mathrm{BmN}$ and $\mathrm{Sf}-9$ cells was successful.

Screening and identification of transgenic silkworms: The transgenic vector was injected into five copulatory pouches of copulated female moths. The eggs were incubated at $25^{\circ} \mathrm{C}$. Green fluorescence could be observed in some eggs (Fig. 2a and b) indicating the introduction of exogenous DNA into these eggs. There were 11 pupae (about $0.44 \%$ ) with green fluorescence among the GO transgenic silkworm generation (Fig. 2c and d). The fluorescent silkworms were allowed to develop into moths, copulate with other fluorescent moths and lay eggs for seed reservation. Genomic DNA was extracted from the copulated moths and subjected to PCR amplification of the $h G M-C S F$ gene using the primer pairs hGM-CSF-1/hGM-CSF-2. The specific $h G M-C S F$ gene product (about $0.43 \mathrm{~kb}$ ) was successfully amplified from the genomes of the fluorescent moths. Likewise, the specific PCR product representing the $h G M-C S F$ gene was detected in the genome of fluorescent silkworms of generations G1-G6 (Fig. 2e). In addition to PCR identification, a G5-generation fluorescent silkworm with the hGM-CSF expression cassette was identified by dot hybridization using a DIG-labeled gfp probe (Fig. 2f). As expected, the GFP probe specifically hybridized with the genome of this G5 fluo rescent moth, indicating that the $g f p$ gene had been integrated into the genome.

Expression of hGM-CSF in transformed $\mathrm{BmN}$ and $\mathrm{Sf}-9$ cells and in transgenic silkworms: Transformed cells showed green fluorescence and survived in zeocincontaining medium demonstrating that the zeocin-gfp 

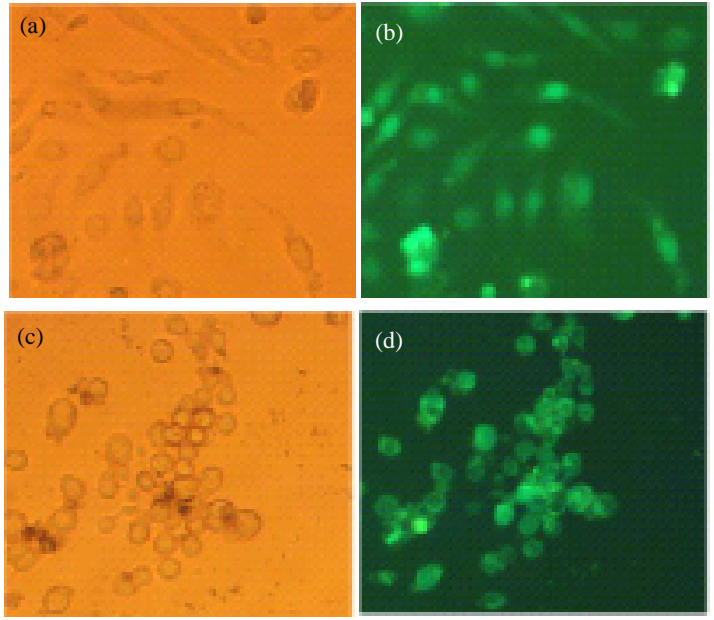

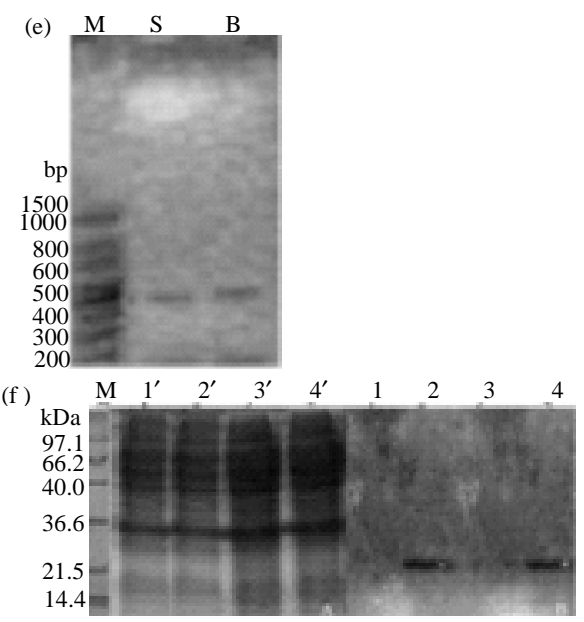

Fig. 1a-d): Screening and identification of transgenic BmN and Sf-9 cells. Transformed cells screened from BmN and Sf-9 cells transfected with pIZT-E-hGM-CSF using zeocin. a) Transformed BmN cells viewed under natural light; b) Transformed BmN cells viewed under fluorescent light; c) Transformed Sf-9 cells viewed under natural light; d) Transformed Sf-9 cells viewed under fluorescent light and e) PCR identification of hGM-CSF. M: DNA marker, S: PCR product of transformed Sf-9 cell genome, B: PCR product of transformed BmN cell genome. f) SDS-PAGE and Western blot analysis of hGM-CSF in cells. f) SDS-PAGE; Lane 1: protein from normal insect Sf-9 cells, Lane 2: protein from stably-transformed insect Sf-9 cells, Lane 3: protein from normal silkworm BmN cells, Lane 4: protein from stably-transformed silkworm BmN cells. B: Western blot; Lanes 1'-4' correspond to Lane 1-4
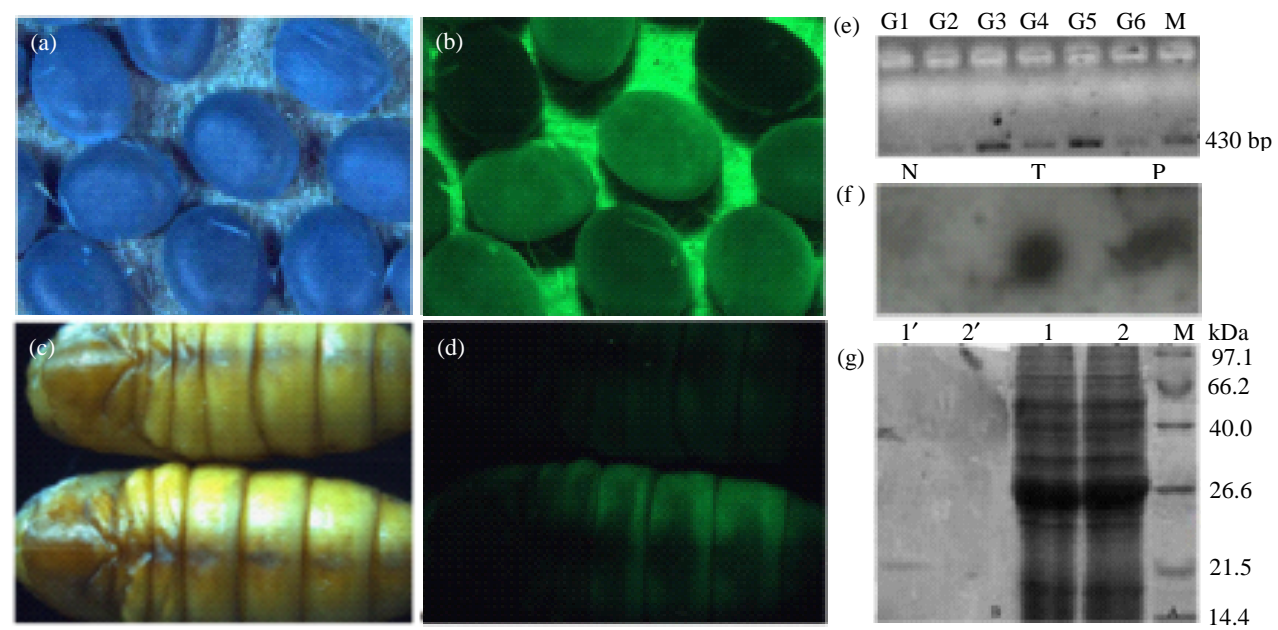

Fig. 2a-d): Screening and identification of transgenic silkworm. Fluorescence of transformed silkworm eggs and pupae. a) Eggs viewed under natural light; b) Eggs viewed under fluorescent light; c) Pupae viewed under natural light; d) Pupae viewed under fluorescent light and e) Identification of G5-generation fluorescent silkworms with hGM-CSF by dot hybridization with a DIG-labeled gfp probe. N: wild-type silkworm genome, T: transgenic silkworm genome, P: positive control group. f) PCR identification of hGM-CSF in transformed silkworm genomes from different generations. Lanes G1-G6: hGM-CSF PCR products from genomes of G1-G6 transgenic silkworms, M: DNA marker. g) SDS-PAGE and Western blot analysis of hGM-CSF in PSGs of transgenic silkworms. A: SDS-PAGE, Lane 1: PSG protein from transformed silkworms, Lane 2: PSG protein from normal silkworms. B: Western blot, Lanes 1' and 2' correspond to Lanes 1 and 2 
fusion gene was expressed in these cells. To examine the expression of the $h G M-C S F$ gene, transformed cells were collected after 3 months of screening and subjected to SDS-PAGE and Western blotting. No specific protein bands representing zeocin-GFP or hGM-CSF were detected by SDS-PAGE but Western blotting using an antibody against hGM-CSF identified a specific band with a molecular weight of $22 \mathrm{kDa}$ (Fig. 1f). The expression levels of hGM-CSF in the transformed cells after 3 months of screening, estimated by ELISA were approximately $0.7 \mathrm{ng}$ in $10^{6} \mathrm{BmN}$ cells and $0.3 \mathrm{ng}$ in $10^{6} \mathrm{Sf}-9$ cells. In contrast, no hGM-CSF expression was detected in normal BmN and Sf-9 cells.

Likewise, a specific band representing hGM-CSF was detected in the PSGs of G5 transgenic silkworms by western blotting but not in PSGs from normal silkworms (Fig. 2g). The expression level of hGM-CSF in freeze-dried silk glands of transgenic silkworms was approximately $4.7 \mathrm{ng} \mathrm{g}^{-1}$.

\section{DISCUSSION}

Insect cell systems are widely used to produce proteins because insect cells have similar patterns of and capacities for co-translational and post-translational modifications such as glycosylation, phosphorylation and protein processing to mamma lian cells. There are two commonly-used strategies for transforming insect cells; one uses an insect BEVS and the other uses transposable elements such as piggyback which is a Class II movable component, initially isolated from a Trichoplusiani cell line. The functions of the piggyBac transposon have been identified previously and developed into an important insect embryo transformation system (Handler et al., 1998).

The pIZT/V5-His vector was developed by Invitrogen for the stable expression of exogenous genes in Sf-9 insect cells. This vector employs the IE2 promoter from OpNPV to control exogenous gene expression. Moreover, the addition of a zeocin-resistance marker makes it easy to screen and confirm the integration of the exogenous gene into the host cell genome. Stablytransformed $\mathrm{BmN}$ cells could be generated by transfection with pIZT-hGM-CSF indicating that the IE promoter of OpNPV retains its promotion activity in silkworm $\mathrm{BmN}$ cells and suggesting that the pIZT/V5-His vector could also be utilized to produce transgenic silkworm cells and individuals. Zeocin selection has been associated with high back ground levels in non-transformed $\mathrm{BmN}$ cells (Lu et al., 2011). Researchers also observed that about $20 \%$ of BmN and Sf-9 cells remained alive after treatment with zeocin for 4 weeks but displayed no green fluorescence. However, researchers did not select nontransfected cells for zeocin resistance and therefore cannot exclude the presence of possible endogenous resistance factors in $\mathrm{BmN}$ cells. These results suggest that the $g f p$ gene was not expressed in the non-fluore scent cells that exhibited zeocin resistance because the complete gfp expression cassette was not incorporated into the genomes of the stably-transformed cells. This suggests that the exogenous DNA was randomly integrated into the genomes of the cultured cells. Either the copy number of the zeocin-gfp fused gene differed among the cell ge nomes or the $g f p$ gene was located at different sites in the genome producing different intensities of green fluorescence in the stably-transformed cells. Enhancer trap effects might have facilitated the strong expression of the zeocin-GFP recombinant protein. Novel enhancers or silencers may be discovered by analyzing the genomic sequences where the exogenous DNA was inserted.

Although, the transformed cells displayed green fluorescence and zeocin resistance indicating that the gfp and zeocin-resistance genes had been expressed, no specific bands were detected by SDS-PAGE. However, the expression levels of hGM-CSF in the stably pIZT-hGMCSF-transformed cell lines were approxima tely $0.7 \mathrm{ng} / 10^{6}$ $\mathrm{BmN}$ cells and $0.3 \mathrm{ng} / 10^{6} \mathrm{Sf}-9$ cells which were two orders of magnitude lower than the level observed in the BEVS. This was likely because of the characteristics of the transformed cells and also because the IE2 promoter is not a strong promoter. The expression levels of hGM-CSF in the current study might have been too low to be detected by SDS-PAGE. However, notable differences in fluorescence intensities were detected among the transformed cells suggesting that the levels of gfp expression may differ among individual transformed cells and that a transformed cell line with high levels of exogenous gene expression might be obtained by cell sorting (Farrell et al., 1998).

Transgenic silkworms could be generated by transposon-derived vector mediation and the most widely used such vector in transgenic silkworms is piggyBac (Takahiro et al., 2006; Hino et al., 2006; Ogawa et al., 2007; Kurihara et al., 2007; Zhao et al., 2009) and hGMCSF. The effectiveness of site-specific integration using a targeting vector has been confirmed previously (Yamao et al., 2009; Wu and Cao, 2004). The current study explored the integration of a target gene into the silkworm genome, mediated by a non-transposon and non-targeting vector. After introduction of vector into silkworm eggs by sperm-mediated gene transfer, $0.44 \%$ of G0 silkworms in emitted green fluorescence and the specific PCR product representing the $h G M-C S F$ gene could be 
amplified from the genomic DNA extracted from G0-G6 silkworms. Moreover, the $g f p$ gene could be detected in G5 silkworms by dot hybridization suggesting that transgenic silkworms could be generated via nontransposon vectors and that exogenous genes integrated into the silkworm genome could be inherited stably.

In this study, the expression of hGM-CSF measured by ELISA stayed at a low level both in vitro and in vivo with the level in the PSGs of transgenic silkworms reached $4.7 \mathrm{ng} \mathrm{g}^{-1}$ in freeze-dried silk land powder. This low level might have been the result of non-secretory expression. In addition, protein expression levels are known to be related to mRNA stability and the average half-life of mRNA in rapidly growing eukaryotic cells is approximately $3 \mathrm{~h}$, though the half-life can be dramatically extended in some highly terminally differentiated cells. Fibroin mRNA for example can remain in the silkworm silk gland for 4 days (Zhu et al., 2004). It is therefore possible that the low hGM-CSF expression levels in the current study may have been attributed to its short mRNA halflife both in vitro and in vivo (Zhao et al., 2009; Xue et al., 2011). This would suggest that maintaining or improving the stability of hGM-CSF mRNA in transgenic silkworms might improve its expression. Another possible strategy for improving the expression level would be to optimize the promoter sequence; a recent study showed that optimization of the TATA box region effectively increased target-gene expression in transgenic silkworms (Tatematsu et al., 2010).

Fusion of exogenous genes with the fibroin gene has recently been the most widely used method for expressing exogenous genes in the silk glands of silkworms (Hino et al., 2006; Kurihara et al., 2007; Fraser et al., 1996; Takahiro et al., 2006; Ogawa et al., 2007; Tomita et al., 2003; Yamao et al., 2009; Wu and Cao, 2004), though this often resulted in affected gene activity and low levels of expressed gene products (Hino et al., 2006; Kurihara et al., 2007; Daubnerova et al., 2009). However, Xue et al. (2011) found that although, hGM-CSF expression was low in the silk glands of targeted silk worms (2.70 $\mathrm{ng} \mathrm{g}^{-1}$ offreeze-dried powdered PSG), levels were still sufficient to significantly increase the leukocytecount when administrated intragastrically to cyclo phosphamide-treated mice. Silkworms are edible suggesting that hGM-CSF-transgenic silkworms might be a potential source of medicine suitable for oral administration.

\section{CONCLUSION}

In this study, the molecular weight of hGM-CSF expressed in both transgenic cells and the PSGs of transgenic silkworms (approximately $22 \mathrm{kDa}$ ) was slightly larger than its theoretical size $(16.3 \mathrm{kDa})$ but it was equivalent to the molecular weight of recombinant hGMCSF expressed in host cells transfected with recombinant baculo virus (Gong et al., 2005). This suggests that the hGM-CSF-expression product was modified during the post-translation process.

\section{ACKNOWLEDGEMENTS}

Researchers gratefully acknowledge the financial support of the National Basic Research Program of China (973 Program, 2012CB114605), the National Natural Science Foundation of China (30571404, 30671590), the Natural Science Foundation of Jiangsu Province (BK 2009117) and the Key Fostering Project for Application Research of Soochow University (Q3134991) and a project funded by the Priority Academic Program Development of Jiangsu Higher Education Institutions.

\section{REFERENCES}

Aua, L., T. Liu, B, H. Shena, K. Chooa and S. Wang, 1996. Secretory production of bioactive recombinant human granulocyte-macrophage colony-stimulating factor by a baculovirus expression system. J. Nal. Bio. Technol., 51: 107-113.

Burgess, A., C. Begley, G. Johnson, A. Lopez and W. Mson et al., 1987. Purification and properties of bacterially synthesized human gr anulocytemacrophage colony-stimulating factor. Blood, 69: 43-51.

Cao, G., R. Xue and W. Shen, 2006. Research on hGMCSF transgentic silkworm with piggybac transposon. Sericulture Sci., 32: 324-327.

Cui, L., R. Xue, Y. Lu, G. Cao and C. Gong, 2011. Expression of hIL-28A in transgenic silkworm mediated by non-transposon vector. Prog. Biochem. Bio. Phys., 38: 724-729.

Daubnerova, I., L. Roller and D. Zitoan, 2009. Transgenesis approaches for functional analysis of peptidergic cells in the silk worm Bombyx mori. Gen. Comp. Endocrinol., 162: 36-42.

Farrell, P., M. Lu, J. Prevost, C. Brown, L. Behie and K. Iatrou, 1998. High-level expression of secreted glycoproteins in transformed lepidopteran insect cells using a novel expression vector. Bio. Technol. Bio. Eng., 60: 656-663.

Fraser, M., T. Clszczon, T. Elick and C. Bauser, 1996. Precise excision of TTAA-specific lepidopteran transposons piggy Bac (IFP2) and tagalong (TFP3) from the baculovirus genome in cell lines from two species of Lepidoptera. Insect Mol. Biol., 5: 141-151. 
Gong, C., R. Xue, G. Cao, X. Shi and W. Shen, 2005. hGMCSF expression by using recomb in ant bmnpv with inactivated chitinase and cathepsin genes. Prog. Biochem. Biophys., 32: 947-952.

Grossmann, M., R. Wong, N. The, J. Tropea, J. EastPalmer, B. Weintraub and M. Szkudlinski, 1997. Expression of biologically active human thyrotropin (hTSH) in a baculovirus system, Effec t of insect cell glycosylation on hTSH activity in vitro and in vivo. Endocrinology, 138: 92-100.

Handler, A., S. Mc Combs and M. Fraser, 1998. The lepidopteran transposon vector, piggyBac, mediates germ-line transformation in the Mediterranean fruit fly. Proc. Nat. Acad. Sci. USA, 95: 7520-7525.

Harrison, R. and D. Jarvis, 2007. Transforming lepidopteran insect cells for improved protein processing. Methods Mol. Biol., 388: 341-356.

Henderson, J., A. Atkinson, C. Lazarus, C. Hawes, R. Napier, H. Macdonald and L. King, 1995. Stable expression of maize auxin binding protein in insect cell lines. Fed. Eur. Biochem. Soc. Lett., 371: 293-296.

Hino, R., M. Tomita and K. Yoshizato, 2006. The generation of germline transgenic silk worms for the production of biologic ally active recombinant fusion proteins of fibroin and human basic fibroblast growth factor. Bio. Mater., 27: 5715-5724.

Jarvis, D. and E. Finn, 1996. Modifying the insect cell $\mathrm{N}$-glycosylation pathway with immediate early baculovirus expression vectors. Nat. Bio. Technol., 14: 1266-1292.

Jarvis, D., J. Flemina, G. Kovacs, M. Summers and L. Guarino, 1990. Use of early baculovirus promoters for continuous expression and efficient processing of foreign gene products in stably transformed lepidopteran insect cells. Biotechnology, 6: 950-955.

Joyce, K., A. Atkinson, I. Bermudez, D. Beadle and L. Kino, 1993. Synthesis of functional GABA-A receptors in stable insect cell lines. Fed. Eur. Biochem. Soc. Lett., 335: 61-64.

Kodaira, K., R. Takahashi, M. Hirabayashi, T. Suzuki and M. Ueda, 1997. Recombinant human GM-CSF in the milk of transgenic rats. Theriogenology, 47: 216-216.

Kurihara, H., H. Sezutsu, T. Tamura and K. Yamada, 2007. Production of an active feline interferon in the cocoon of transgenic silk worms using the fibroin $\mathrm{H}-$ cha in expression system. Biochem. Biophys. Res. Commun., 355: 976-980.

Lu, Y., X. Zheng, R. Xue, G. Cao, W. Shen and C. Gong, 2011. Antip roliferative activity of recombinant human interferon-e 2 expressed in stably transformed BmN cells. Afr. J. Biotechnol., 10: 7260-7266.
Mandrioli, M. and E. Wimmer, 2003. Stable transformation of a Mamestra brassicae (lepidoptera) cell line with the lepidopteran-derived transposon piggyback. Insect Biochem. Mol. Biol., 33: 1-5.

Miyajima, A., K. Otsu, J. Schreurs, M.W. Bond, J.S. Abrams and K. Arai, 1986. Expression of murine and human granulocyte-macrophage colonystimula ting factors in $S$. cerevisiase, mutagenesis of the potential glycosylation sites. EMBO J., 5: 1193-1197.

Ogawa, S., M. Tomita, K. Shimizu and K. Yoshizato, 2007. Generation of transgenic silk worm that secretes recombinant proteins in the seric in layer of cocoon, Production of recombinant human serum albumin. J. Biotechnol., 128: 531-544.

Ryoo, Z., M. Kim, K. Kim, Y. Bahk and J. Lee et al., 2001. Expression of recombinant human granulocyte macrophage-colony stimulating factor (Hgm-CSF) in mouseurine. Trans. Res., 10: 193-200.

Shi, X., J. Qin, J. Zhu and D. Zhu, 1996. Expression of biologically active human granulocyte-macrophage colony-stimulating factor in the silkworm (Bombyx mori). Applied Biochem. Biotechnol., 24: 245-249.

Takahiro, A., M. Tomita, K. Shimizu, S. Ogawa and K. Yoshizato, 2006. Generation of hybrid transgenic silkworms that express Bombyx mori prolyl-hydroxyla sea-subunits and human collagens in posterior silk glands, Production of cocoons that contained collagens with hydroxylated praline residues. J. Biotechnol., 126: 205-219.

Tamura, T., C. Thibert, C. Royer, T. Kanda and A. Eappen et al., 1997. Bovine alpha s1-casein gene sequences direct high level expression of human granulocyte-macrophage colony-stimulating factor in the milk of transgenic mice. Trans. Res., 6: 75-84.

Wickham, T., T. Davis, R. Granados, M. Shuler and H. Wood, 1992. Screening of insect cell lines for the production of recombinant proteins and infectious virus in the baculovirus expression systems. Biotechnol. Bioeng., 8: 391-396.

Wong, G., J. Witek, P. Temple, K. Wilkens and A. Leary et al., 1985. Human GM-CSF, Molecular cloning of the complementary DNA and purification of the natural and recombinant protein. Science, 228: 810-815.

$\mathrm{Wu}, \mathrm{X}$. and C. Cao, 2004. Targeting of human a FGF gene into silk worm, Bombyx mori L. through homologous recombination. J. Zhejiang Univ. Sci., 5: 644-650. 
Xue, R., H. Chen, L. Cui, G. Cao, W. Zhou, X. Zheng and C. Gong, 2011. Expression of hGM-CSF in silk glands of transgenic silkworms using gene targeting vector. Transgen. Res., 21: 101-111.

Xue, R., X. Li, Y. Zhao, X. Pan, X. Zhu, G. Cao and C. Gong, 2009. Elementary research into the transformation $\mathrm{BmN}$ cells mediated by the piggyBac transposon vector. J. Biotechnol., 144: 272-278.

Yamao, M., N. Katayama, H. Nakazawa, M. Yamakawa and Y. Hayashi et al., 2009. Gene targeting in the silk worm by use of a baculovirus. Gen. Dev., 13: 511-516.
Zhang, J., N. Kalogerakis, L. Behie and K. Iatrou, 1993. A two-stage bioreactor system for the production of recombinant proteins using a genetically engineered baculovirus/insect cell system. Biotechnol. Bioeng., 42: $357-366$.

Zhao, Y., X. Li and G. Cao, 2009. Expression of hIGF-1 in the silk glands of transgenic silk worms and in transformed silkworm cells. Sci. China Ser. C, Life Sci., 39: 677-684.

Zhu, Y., Y. Li and X. Zhen, 2004. Modern Molecular Biology. 2nd Edn., High Education Press, Beijing, Pages: 288. 